The impressive but sad list of over forty clinical studies using various cytotoxic chemotherapies published in the last few years has failed to increase median survival of glioblastoma beyond two years after diagnosis. In view of this apparent brick wall, adjunctive non-cytotoxic growth factor blocking drugs are being tried, as in the CUSP9* protocol. A related theme is searching for agonists at growth inhibiting receptors. One such dataset is that of melatonin agonism at M1 or M2 receptors found on glioblastoma cells, being a negative regulator of these cells' growth. Melatonin itself is an endogenous hormone, but when used as an exogenously administered drug it has many disadvantages. Agomelatine, marketed as an antidepressant, and ramelteon, marketed as a treatment for insomnia, are currently-available melatonin receptor agonists. These melatonin receptor agonists have significant advantages over the natural ligand: longer half-life, better oral absorption, and higher affinity to melatonin receptors. They have an eminently benign side effect profile. As full agonists they should function to inhibit glioblastoma growth, as demonstrated for melatonin. A potentially helpful ancillary attribute of melatonergic agonists in glioblastoma treatment is an increase in interleukin-2 synthesis, expected, at least partially, to reverse some of the immunosuppression associated with glioblastoma.

Key words: agomelatine, CUSP9*, glioblastoma, interleukin-2, melatonin, ramelteon, temozolamide.

Contemp Oncol (Pozn) 2015; 19 (2): 157-162 DOI: $10.5114 /$ wo.2015.51421

\section{Agomelatine or ramelteon as treatment adjuncts in glioblastoma and other M1- or M2-expressing} cancers

\author{
Richard E. Kast
}

IIAIGC Study Center, USA

Preamble: an orthopaedic aphorism:

If a little force doesn't work, maybe more force will.

\section{Introduction}

This paper presents some physiological data supporting adjunctive use of agomelatine or ramelteon in our ongoing effort to improve glioblastoma treatment, given that the current median overall survival remains less than two years [1]. An impressive but sad list of over forty clinical studies, using various cytotoxic chemotherapies and published in the last few years [1, 2], has failed to increase the median survival of glioblastoma beyond two years after initial diagnosis. Many of these studies imposed high side-effect burdens on patients. To redress this situation we took a new approach with the CUSP9* protocol $[1,2]$. In CUSP9*, repositioned older drugs, drugs that have not traditionally been used in cancer chemotherapy, are used to block 17 different survival paths used by glioblastoma cells to survive, grow, and avoid cytotoxicity from traditional cytotoxic chemotherapy drugs. We now present past data and explain the rationale to indicate the potential for the benefit of the addition of currently marketed melatonin agonists agomelatine or ramelteon as adjuncts to a compatible, coordinated polypharmaceutical regimen during glioblastoma treatment.

\section{Nile Distributary Problem}

Recent oncology studies and thinking, both generally and regarding glioblastoma specifically [1-3], have drawn the conclusion that simultaneous blocking of multiple, cross-covering growth paths will be required for meaningful clinical benefit. This has been termed the Nile Distributary Problem (Fig. 1) [expanded in refs. 1 and 2]. Multiple cross-covering growth stimulating or growth enabling pathways imply a] non-existence of a single or crucial nodal flaw, and b] the consequent need of wide-net polypharmacy. A growth stimulating signalling system that we should inhibit and a growth inhibiting system that we should stimulate are considered functionally equivalent for the purposes of this discussion.

It is a widely implemented principle of modern engineering that if a single part or function of a well-engineered machine were to fail and result in catastrophic or significant harm, that part or function must be made redundant, often several times redundant. For example, many modern jet airliners would become unflyable without their computer's help. Thus they have four redundant computers, any one of which can safely allow the plane to fly. After a thousand million years mammalian cells have become well-engineered entities and therefore have multiple redundant systems for crucial functions. Many of these go simultaneously awry in cancer, implying a need for multiple simultaneous interventions. The Nile Distributary Problem. 


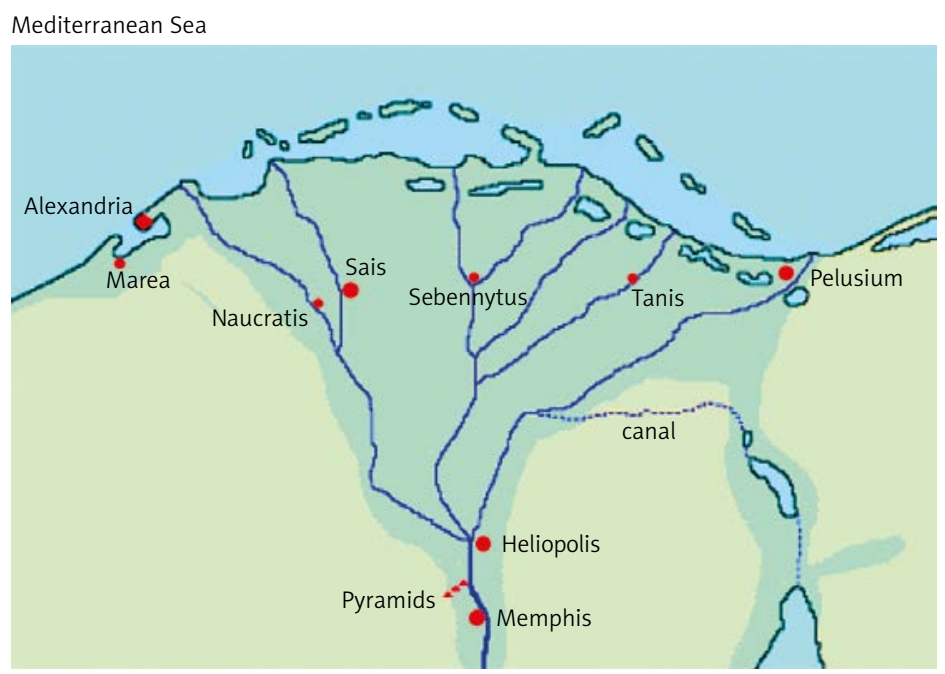

Fig. 1. The Nile Distributary Problem. Above is a drawing of the major Nile River distributaries. There are dozens of minor distributaries (not shown) that could become major ones and take up the extra flow created by a blockage in a major distributary. One can imagine that blocking one or a few of the many distributaries would have little effect on total flow to the sea. In the same way, we can view the many recent failures to clinically benefit from profound pharmacological blocking of a single growth path, even when that path had been known to be active in, and a major driver of, glioblastoma growth [1]

In light of and in recognition of the Nile Distributary Problem, agomelatine or ramelteon as treatment adjuncts during glioblastoma treatment are suggested only as a component of any larger CUSP-like regimen, not as a stand-alone option. According to this understanding, clinical control or cure will come when enough of the cross-covering growth paths are blocked so that glioblastoma cell survival is seriously impaired. To that end, this paper now reviews evidence for the role of agonism at the two currently recognised, widely expressed melatonin receptors, $\mathrm{M} 1$ and $\mathrm{M} 2$, in glioblastoma growth inhibition, and the potential for either of these two commercially approved and marketed M1/M2 agonists to enhance M1/M2-mediated growth inhibition - a counterweight to growth-stimulating paths.

\section{Melatonin as a negative regulator of glioblastoma growth}

\section{Melatonin}

Melatonin (N-acetyl-5-methoxytryptamine) is a $232 \mathrm{Da}$, short half-life hormone [4, 5] synthesised downstream

Table 1. Circulating half-life data for melatonergic drugs. All three drugs are available for use in humans. T1/2 is the circulating half-life

$\begin{array}{lccc}\text { Molecule } & \text { T 1/2 } & \text { Animal } & \text { Reference } \\ \text { Agomelatine } & 2 \text { hours } & \text { human } & 13 \\ \text { Ramelteon } & \sim 1-2 \text { hours } & \text { human } & 67 \\ \text { Ramelteon } & \sim 1-2 \text { hours } & \text { human } & 52 \\ \text { Melatonin } & 23 \text { min } & \text { rat } & 68 \\ \text { Melatonin } & <20 \text { min } & \text { hamster } & 69 \\ \text { Melatonin } & 40 \text { min } & \text { human } & 42 \\ \text { Melatonin } & \sim 30 \text { min } & \text { human } & 70\end{array}$

from serotonin $[6,7]$ and found universally in mammals [8]. Melatonin signals through two outer surface membrane receptors, termed M1 and M2 [9]. Dietary tryptophan is used either i) in protein synthesis, ii) shunted along the kynurenine pathway, or iii) shunted to serotonin synthesis [10]. From the serotonin path melatonin is synthesised [11]. Melatonin as a pharmacological agent has several drawbacks, very short circulating half-life (Table 1) and erratic absorption [12] among them. Pharmacological melatonergic agonists were developed to circumvent these drawbacks and marketed to help initiate sleep during diurnal dysregulation states or to alleviate depression $[13,14]$.

Traditionally, melatonin has been considered a pineal-synthesised hormone. Melatonin is found throughout vertebrates' body and brain [15], but also in the brain of ancient, primitive life forms such as planaria [16] and marine zooplankton [17]. More recent evidence shows that lymphocytes can synthesise melatonin [18-20], as do other cells involved with innate immunity [21] and inflammation [22]. Normal brain astrocytes and glia also synthesise melatonin [23], as does the gut [24] and other organs [5].

Although best known for diurnal sleep-wake regulation and synthesis in the pineal gland [5], melatonin has significant physiological functions other than sleep-wake cycling $[9,25]$.

Multiple potential areas for melatonin's anti-cancer action have been postulated by others [25-29]. Detailed below are data on melatonin function specifically in glioblastoma growth.

\section{Melatonin and glioblastoma}

The concept of glioblastoma stem cells does not have a precise definition yet, and it may never have one single such definition if, as many now suspect, stemness comprises a range of somewhat differing attributes in subsets of cells, not a unitary entity [30]. Within various 
current concepts of glioblastoma, stem cells generally encompass four core cell attributes: i) relative quiescence, ii) a sub-population from which post-chemotherapy (irradiation, resection) glioblastoma preferentially re-grows, iii) enhanced in vitro clonogenicity where, relative to the non-stem population, fewer cells are required to establish three dimensional growth in soft agar, and iv] they can divide symmetrically or asymmetrically to both stem or nonstem daughter cells [3, 31-33].

Melatonin-whether by agonism at $\mathrm{M} 1$ or $\mathrm{M} 2$ or by both is unclear-empirically lowers glioblastoma cell proliferation generally and the stem sub-population plating efficiency (clonogenicity) specifically [34]. The mode of glioblastoma stem sub-population death is autophagy [34]. Melatonin alone shows cytotoxicity to two glioma cell lines that also lower $I C_{50}$ of temozolamide by 3 to 6 fold [35]. Temozolamide is currently the mainstay cytotoxic drug in treating glioblastoma $[3,36]$. Under either hypoxia or normoxia, melatonin slows centrifugal migration of glioblastoma cell lines [37]. This would be of considerable benefit were this to hold in vivo and in human disease, given that it is precisely these post-primary resection centrifugally migrating cells that go on to kill patients.

A remarkable study from 1996 that does not seem to have been followed up showed that of glioblastoma patients given 60 Gy irradiation plus 20 mg melatonin orally daily for 1 year, 6 of 14 were alive, while in the control group, given 60 Gy alone, only 1 of 16 was alive at 1 year [38]. Two years later (in 1998) this same group published a study in a group of various advanced stage cancers claiming survival benefit from melatonin $20 \mathrm{mg}$ orally per day plus an aloe extract [39]. Procedural study weaknesses and non-replication by others prevent analysis of this data. Still, miraculous and unrefuted results, over a decade old and neither replicated nor followed up, neither widely instituted or acknowledged, should not, cannot, be assumed just on that basis to be incorrect.

\section{Agomelatine}

Agomelatine is a 243 Da melatonin agonist licensed by the EMA (but not the FDA) for treating depression [40]. It has a higher affinity to both $M 1$ and $M 2$, with a pKi of about $10 \mathrm{nM}$, than does the natural ligand and longer half-life about two hours $[4,14,41]$, see Table 1 . The evanescent nature of any circulating melatonin has been previously noted as a clinical problem [4], an impediment to effective treatment using exogenous melatonin itself. Added disadvantages include melatonin's poor and erratic oral bioavailability [12] with correspondingly highly variable blood levels in subjects given identical doses [42].

Agomelatine is marketed as an antidepressant with dual modes of action: a) agonism at M1 and M2 melatonin receptors and and $b$ ) antagonism at serotonin 2 C receptors (5-HT2C) [43-46]. It readily penetrates the blood-brain barrier. The status or efficacy of agomelatine as an antidepressant is uncertain as of spring 2015, but its excellent tolerability is unequivocal [41] as is its ability to improve sleep during a major depression [47, 48], indicative of potent M1/M2 agonism.

\section{Ramelteon}

Ramelteon is a $259 \mathrm{Da}$. brain-penetrant melatonin agonist, FDA approved [but not approved by the EMA] to help sleep initiation $[49,50]$. It is remarkably free of side effects [49-51], in part because of receptor agonism limited to M1 and $M 2$ melatonin receptors. Also of note, ramelteon affinity to $M 1$ and $M 2$ exceeds that of melatonin itself by an order of magnitude, and oral absorption is good. Circulating half-life of ramelteon is several times longer than that of melatonin [52], see Table 1.

\section{Augmenting the augmenter}

\section{Increasing ramelteon exposure}

Ramelteon is metabolised primarily by hepatic CYP 1 A2 $[53,54]$. The antidepressant serotonin reuptake inhibitor fluvoxamine is one of the most potent inhibitors known of CYP $1 A 2$ and empirically is found to increase circulating ramelteon levels $>100$ fold $[54,55]$, thus giving us the potential to strongly stimulate $M 1$ and $M 2$ receptors on glioblastoma cells, augmenting the augmentation ramelteon might offer when added to other glioblastoma treatments.

\section{Interleukin 2 and melatonin receptors}

Interleukin-2 (IL-2) is a $14 \mathrm{kDa}$ protein that contributes to driving $T$ lymphocyte clonal expansion $[56,57]$. Exogenous IL-2 is the first safe and effective pharmacological agent augmenting immunological anti-cancer effects, often giving complete regression in metastatic melanoma and renal cancer [56-58]. Interleukin-2 is in active study for use in other cancers [59].

The wide array of proposed mechanisms by which melatonin might exert its anti-cancer effects have been reviewed elsewhere [60]. Increasing IL-2 might be one of them. Lymphocytes bear melatonin receptors [61]. Of central importance, melatonin exposure enhances lymphocyte's IL-2 synthesis $[62,63]$. Empirically, oral melatonin, $20 \mathrm{mg}$ per day given to hepatocellular patients along with transarterial catheter embolisation, increased circulating IL-2 compared to those receiving embolisation alone [64]. We would expect agomelatine and ramelteon to be even more potent and effective in this regard, given these drugs' higher affinity to melatonin receptors and longer half-life than the natural ligand.

\section{Caveats}

Cancers have multiple dysregulated physiological systems that act together as an ensemble to mediate malignant behaviour. These dysregulated systems are different over time and space and are different in different parts of the same tumour, so single biopsy study will not give an accurate picture of the complete array of dysregulated systems active over time in a particular tumour. Growth driving systems are not stable; they shift. These systems cross-cover for each other when a pathway is blocked by our drug treatment, others easily take over, compensating for the inhibited pathway, implying a requirement for extensive polypharmacy for effective treatment, as in Nile Distributary Problem. 
Several non-oncology drugs with ancillary attributes that inhibit these pathways can be used simultaneously to block enough pathways to stop growth. This requires an admittedly unpleasant extensive polypharmacy. CUSP9* [1] is the ten-drug treatment protocol for recurrent glioblastoma resulting from these considerations.

There is debate as to what degree the antioxidant effect of melatonin is mediated by the molecule itself or intracellular physiological events consequent to $M 1$ or M2 agonism [65, 66]. Since several elements of CUSP9* increase intracellular reactive oxygen species (ROS), and by design such an ROS increase contributes to anti-glioblastoma action, melatonin agonists like agomelatine or ramelteon might best be combined with polypharmaceutical regimens that do not rely on or mediate an increase in intracellular ROS as part of their mechanism of anti-cancer action, until this matter is settled. In other words, melatonin agonists might not be suitable for combination with CUSP9* itself. Given the data on melatonin's potent enhancing of temozolamide cytotoxicity to glioma cell lines [35], adjuvant agomelatine or ramelteon might be best used during initial Stupp Protocol treatment (temozolamide with 60 Gy irradiation) [3].

\section{Conclusions}

The limited progress in treating glioblastoma evident over the last decades prompts us to look beyond the traditional array of cytotoxic drugs for new paths to attack this hardy cancer. To augment the current standard cytotoxic drug in glioblastoma, temozolamide, we have briefly outlined past data showing how glioblastoma cells express both melatonin receptors $M 1$ and $M 2$, the stimulation of which seems to be a negative regulator of glioblastoma cell growth. We have readily available, potent, and well-tolerated $\mathrm{M} 1$ and $\mathrm{M} 2$ agonist drugs agomelatine [EMA approved, marketed in EU] and ramelteon [FDA approved, marketed in the USA]. These melatonin receptor agonists have significant advantages over the natural ligand: longer half-life, better oral absorption, and higher affinity to melatonin receptors.

The Stupp Protocol, maximal feasible resection, irradiation, and classic cytotoxic chemotherapy with temozolamide [3] is the current standard initial treatment for glioblastoma. As outlined, ideally agomelatine or ramelteon would be part of a coordinated polypharmacy. However, at the least, given three prominent facts: i) survival more than two years after diagnosis is still unusual, ii) agomelatine and ramelteon are exceptionally well-tolerated, very low risk medicines, and iii) the outlined data showing melatonergic agonism augments temozolamide cytotoxicity to glioblastoma cells, the risk/benefit of adding agomelatine or ramelteon to standard Stupp Protocol is highly skewed to proceeding to study such addition.

As in the Preamble, adjunctive agomelatine and ramelteon added during an appropriate phase of glioblastoma treatment might be an effective way to apply more force, making a dent in this heretofore intractable disease.

The author declares no conflict of interest.

\section{References}

1. Kast RE, Karpel-Massler G, Halatsch ME. CUSP9* treatment protocol for recurrent glioblastoma: aprepitant, artesunate, auranofin, captopril, celecoxib, disulfiram, itraconazole, ritonavir, sertraline augmenting continuous low dose temozolomide. Oncotarget 2014; 5: 8052-82.

2. Kast RE, Boockvar JA, Bruning A, et al. A conceptually new treatment approach for relapsed glioblastoma: coordinated undermining of survival paths with nine repurposed drugs (CUSP9) by the International Initiative for Accelerated Improvement of Glioblastoma Care. Oncotarget 2013; 4: 502-30.

3. Xie Q, Mittal S, Berens ME. Targeting adaptive glioblastoma: an overview of proliferation and invasion. Neuro Oncol 2014; pii: nou147.

4. Cardinali DP, Srinivasan V, Brzezinski A, Brown GM. Melatonin and its analogs in insomnia and depression. J Pineal Res 2012; 52: 365-75.

5. Barrenetxe J, Delagrange P, Martinez JA. Physiological and metabolic functions of melatonin. J Physiol Biochem 2004; 60: 61-72.

6. Reiter RJ, Tan DX, Galano A. Melatonin: Exceeding Expectations. Physiology (Bethesda) 2014; 29: 325-33.

7. Singh M, Jadhav HR. Melatonin: functions and ligands. Drug Discov Today 2014; 19: 1410-8.

8. Hardeland R. Neurobiology, pathophysiology, and treatment of melatonin deficiency and dysfunction. ScientificWorldJournal 2012; 2012: 640389.

9. Zlotos DP, Jockers R, Cecon E, Rivara S, Witt-Enderby PA. MT1 and MT2 melatonin receptors: ligands, models, oligomers, and therapeutic potential. J Med Chem 2014; 57: 3161-85.

10. Campbell BM, Charych E, Lee AW, Möller T. Kynurenines in CNS disease: regulation by inflammatory cytokines. Front Neurosci 2014; 8: 12.

11. Oxenkrug G. Serotonin-kynurenine hypothesis of depression: historical overview and recent developments. Curr Drug Targets 2013; 14: 514-21.

12. DeMuro RL, Nafziger AN, Blask DE, Menhinick AM, Bertino JS Jr. The absolute bioavailability of oral melatonin. J Clin Pharmacol 2000; 40: 781-4.

13. Hardeland, R. New approaches in the management of insomnia: weighing the advantages of prolonged release melatonin and synthetic melatoninergic agonists. Neuropsychiatr. Dis Treat 2009; 5: 341-54.

14. Hardeland R, Poeggeler B, Srinivasan V, Trakht I, Pandi-Perumal SR, Cardinali DP. Melatonergic drugs in clinical practice. Arzneimittelforschung 2008; 58: 1-10.

15. Acuna-Castroviejo D, Escames G, Venegas C, et al. Extrapineal melatonin: sources, regulation, and potential functions. Cell Mol Life Sci 2014; 71: 2997-3025.

16. Itoh MT, Shinozawa T, Sumi Y. Circadian rhythms of melatonin-synthesizing enzyme activities and melatonin levels in planarians. Brain Res 1999; 830: 165-73.

17. Tosches MA, Bucher D, Vopalensky P, Arendt D. Melatonin signaling controls circadian swimming behavior in marine zooplankton. Cell 2014; 159: 46-57.

18. Carrillo-Vico A, Calvo JR, Abreu P, Lardone PJ, Garcia-Maurino S, Reiter RJ, Guerrero JM. Evidence of melatonin synthesis by human lymphocytes and its physiological significance: possible role as intracrine, autocrine, and/or paracrine substance. FASEB J 2004; 18: 537-9.

19. Carrillo-Vico A, Lardone PJ, Fernandez-Santos JM, Martin-Lacave I, Calvo JR, Karasek M, Guerrero JM. Human lymphocyte-synthesized melatonin is involved in the regulation of the interleukin-2/ interleukin-2 receptor system. J Clin Endocrinol Metab 2005; 90: 992-1000.

20. Lardone PJ, Guerrero JM, Fernandez-Santos JM, Rubio A, Martin-Lacave I, Carrillo-Vico A. Melatonin synthesized by T lymphocytes as a ligand of the retinoic acid-related orphan receptor. J Pineal Res 2011; 51: 454-62.

21. Calvo JR, Gonzalez-Yanes C, Maldonado MD. The role of melatonin in the cells of the innate immunity: a review. J Pineal Res 2013; 55: 103-20. 
22. Chen $\mathrm{O}$, Wei W. Effects and mechanisms of melatonin on inflammatory and immune responses of adjuvant arthritis rat. Int Immunopharmacol 2002; 2: 1443-9.

23. Tan DX, Manchester LC, Sanchez-Barcelo E, Mediavilla MD, Reiter RJ. Significance of high levels of endogenous melatonin in Mammalian cerebrospinal fluid and in the central nervous system. Curr Neuropharmacol 2010; 8: 162-7.

24. Chen CQ, Fichna J, Bashashati M, Li YY, Storr M. Distribution, function and physiological role of melatonin in the lower gut. World J Gastroenterol 2011; 17: 3888-98.

25. Srinivasan V, Pandi-Perumal SR, Brzezinski A, Bhatnagar KP, Cardinali DP. Melatonin, immune function and cancer. Recent Pat Endocr Metab Immune Drug Discov 2011; 5: 109-23.

26. Cutando A, Lopez-Valverde A, Arias-Santiago S, DE Vicente J, DE Diego RG. Role of melatonin in cancer treatment. Anticancer Res 2012; 32: 2747-53.

27. Bizzarri M, Proietti S, Cucina A, Reiter RJ. Molecular mechanisms of the pro-apoptotic actions of melatonin in cancer: a review. Expert Opin Ther Targets 2013; 17: 1483-96

28. Rondanelli M, Faliva MA, Perna S, Antoniello N. Update on the role of melatonin in the prevention of cancer tumorigenesis and in the management of cancer correlates, such as sleep-wake and mood disturbances: review and remarks. Aging Clin Exp Res 2013; 25: 499-510.

29. Zarogoulidis P, Darwiche K, Huang H, Spyratos D, Yarmus L, Li Q, Kakolyris S, Syrigos K, Zarogoulidis K. Time recall; future concept of chronomodulating chemotherapy for cancer. Curr Pharm Biotechnol 2013; 14: 632-42.

30. Antoniou A, Hebrant A, Dom G, Dumont JE, Maenhaut C. Cancer stem cells, a fuzzy evolving concept: a cell population or a cell property? Cell Cycle 2013;12: 3743-8.

31. Bayin NS, Modrek AS, Placantonakis DG. Glioblastoma stem cells: Molecular characteristics and therapeutic implications. World J Stem Cells 2014; 6: 230-8.

32. Kast RE, Belda-Iniesta C. Suppressing glioblastoma stem cell function by aldehyde dehydrogenase inhibition with chloramphenicol or disulfiram as a new treatment adjunct: an hypothesis. Curr Stem Cell Res Ther 2009; 4: 314-7.

33. Binda E, Reynolds BA, Vescovi AL. Glioma stem cells: turpis omen in nomen? (The evil in the name?). J Intern Med 2014; 276: 25-40.

34. Martín V, Sanchez-Sanchez A, Puente-Moncada N, Gomez-Lobo M, Alvarez-Vega MA, Antolín I, Rodriguez C. Involvement of autophagy in melatonin-induced cytotoxicity in glioma-initiating cells. J Pineal Res 2014; 57: 308-16.

35. Martín V, Sanchez-Sanchez AM, Herrera F, Gomez-Manzano C, Fueyo J, Alvarez-Vega MA, Antolín I, Rodriguez C. Melatonin-induced methylation of the ABCG2/BCRP promoter as a novel mechanism to overcome multidrug resistance in brain tumour stem cells. Br J Cancer 2013; 108: 2005-12.

36. Wilson TA, Karajannis MA, Harter DH. Glioblastoma multiforme: State of the art and future therapeutics. Surg Neurol Int 2014; 5: 64

37. Zhang Y, Liu Q, Wang F, et al. Melatonin antagonizes hypoxia-mediated glioblastoma cell migration and invasion via inhibition of HIF-1 $\alpha$. J Pineal Res 2013; 55: 121-30.

38. Lissoni P, Meregalli S, Nosetto L, Barni S, Tancini G, Fossati V, Maestroni $G$. Increased survival time in brain glioblastomas by a radioneuroendocrine strategy with radiotherapy plus melatonin compared to radiotherapy alone. Oncology 1996; 53: 43-6.

39. Lissoni P, Giani L, Zerbini S, Trabattoni P, Rovelli F. Biotherapy with the pineal immunomodulating hormone melatonin versus melatonin plus aloe vera in untreatable advanced solid neoplasms. Nat Immun 1998;16: 27-33.

40. Owen RT. Agomelatine: a novel pharmacological approach to treating depression. Drugs Today (Barc) 2009; 45: 599-608.

41. Pompili M, Serafini G, Innamorati M, Venturini P, Fusar-Poli P, Sher L, Amore M, Girardi P. Agomelatine, a novel intriguing antidepressant option enhancing neuroplasticity: a critical review. World J Biol Psychiatry 2013; 14: 412-31.

42. Cavallo A, Ritschel WA. Pharmacokinetics of melatonin in human sexual maturation. J Clin Endocrinol Metab 1996; 81: 1882-6.

43. Koesters M, Guaiana G, Cipriani A, Becker T, Barbui C. Agomelatine efficacy and acceptability revisited: systematic review and meta-analysis of published and unpublished randomised trials. Br J Psychiatry 2013; 203: 179-87.

44. Smeraldi E, Delmonte D. Agomelatine in depression. Expert Opin Drug Saf 2013; 12: 873-80.

45. Hale A, Corral RM, Mencacci C, Ruiz IS, Severo CA, Gentil V. Superior antidepressant efficacy results of agomelatine versus fluoxetine in severe MDD patients: a randomized, double-blind study. Int Clin Psychopharmacol 2010; 25: 305-14.

46. Plesničar BK. Efficacy and tolerability of agomelatine in the treatment of depression. Patient Prefer Adherence 2014; 8: 603-12.

47. Taylor D, Sparshatt A, Varma S, Olofinjana O. Antidepressant efficacy of agomelatine: meta-analysis of published and unpublished studies. BMJ 2014; 348: g1888.

48. Olié JP, Kasper S. Efficacy of agomelatine, a MT1/MT2 receptor agonist with 5-HT2C antagonistic properties, in major depressive disorder. Int J Neuropsychopharmacol 2007; 10: 661-73.

49. Pandi-Perumal SR, Srinivasan V, Spence DW, Moscovitch A, Hardeland R, Brown GM, Cardinali DP. Ramelteon: a review of its therapeutic potential in sleep disorders. Adv Ther 2009; 26: 613-26.

50. McGechan A, Wellington K. Ramelteon. CNS Drugs 2005; 19 : 1057-65.

51. Simpson D, Curran MP. Ramelteon: a review of its use in insomnia. Drugs 2008; 68: 1901-19.

52. Greenblatt DJ, Harmatz JS, Karim A. Age and gender effects on the pharmacokinetics and pharmacodynamics of ramelteon, a hypnotic agent acting via melatonin receptors MT1 and MT2. J Clin Pharmacol 2007; 47: 485-96.

53. Kast RE, Altschuler EL. Co-administration of ramelteon and fluvoxamine to increase levels of interleukin-2. Med Hypotheses 2006; 67: 1389-90

54. Obach RS, Ryder TF. Metabolism of ramelteon in human liver microsomes and correlation with the effect of fluvoxamine on ramelteon pharmacokinetics. Drug Metab Dispos 2010; 38: 1381-91.

55. FDA labelling information [online]. http://www.fda.gov/cder/foi/ label/2005/021782lbl.pdf;2005.

56. Rosenberg SA. IL-2: the first effective immunotherapy for human cancer. J Immunol 2014; 192: 5451-8.

57. Waldmann T, Tagaya Y, Bamford R. Interleukin-2, interleukin-15, and their receptors. Int Rev Immunol 1998; 16: 205-26.

58. McDermott D, Lebbe C, Hodi FS, Maio M, Weber JS, Wolchok JD, Thompson JA, Balch CM. Durable benefit and the potential for long-term survival with immunotherapy in advanced melanoma. Cancer Treat Rev 2014; 40: 1056-1064.

59. Rosalia RA, Arenas-Ramirez N, Bouchaud G, Raeber ME, Boyman O. Use of enhanced interleukin-2 formulations for improved immunotherapy against cancer. Curr Opin Chem Biol 2014; 23C: 39-46.

60. Sanchez-Hidalgo M, Lee M, de la Lastra CA, Guerrero JM, Packham G. Melatonin inhibits cell proliferation and induces caspase activation and apoptosis in human malignant lymphoid cell lines. J Pineal Res 2012; 53: 366-73.

61. Ha E, Han E, Park HJ, et al. Microarray analysis of transcription factor gene expression in melatonin-treated human peripheral blood mononuclear cells. J Pineal Res 2006; 40: 305-11.

62. Garcia-Maurino S, Pozo D, Calvo JR, Guerrero JM. Correlation between nuclear melatonin receptor expression and enhanced cytokine production in human lymphocytic and monocytic cell lines. J Pineal Res 2000; 29: 129-37.

63. Garcia-Maurino S, Gonzalez-Haba MG, Calvo JR, Rafii-El-Idrissi M, Sanchez-Margalet V, Goberna R, Guerrero JM. Melatonin enhances IL-2, IL-6, and IFN-gamma production by human circulating CD4+ cells: a possible nuclear receptor-mediated mechanism involving T helper type 1 lymphocytes and monocytes. I Immunol 1997; 159: 574-81.

64. Yan JJ, Shen F, Wang K, Wu MC. Patients with advanced primary hepatocellular carcinoma treated by melatonin and transcatheter arterial chemoembolization: a prospective study. Hepatobiliary Pancreat Dis Int 2002; 1: 183-6.

65. An Y, Liu E, Liu X, Yang F, Han F, Dai Q. Protective effect of melatonin on neural cells against the cytotoxicity of oxyradicals. Chin Med Sci J 2000; 15: 40-4 
66. Suzen S. Melatonin and synthetic analogs as antioxidants. Curr Drug Deliv 2013; 10: 71-5.

67. Karim A, Tolbert D, Cao C. Disposition kinetics and tolerance of escalating single doses of ramelteon, a high-affinity MT1 and MT2 melatonin receptor agonist indicated for treatment of insomnia. J Clin Pharmacol 2006; 46: 140-8.

68. Gibbs FP, Vriend J. The half-life of melatonin elimination from rat plasma. Endocrinology 1981; 109: 1796-8.

69. Ferreira SA, Rollag MD, Glass JD. Pharmacokinetics of extracellular melatonin in Siberian hamster forebrain. Brain Res 1996; 733 318-20.

70. Mallo C, Zaĭdan R, Galy G, Vermeulen E, Brun J, Chazot G, Claustrat

B. Pharmacokinetics of melatonin in man after intravenous infusion and bolus injection. Eur J Clin Pharmacol 1990; 38: 297-301.

\section{Address for correspondence}

R.E. Kast, MD

IIAIGC Study Center

22 Church Street

Burlington, VT 05401 USA

tel. 802-5577278

richarderickast@gmail.com

Submitted: 7.12.2014

Accepted: $\quad 9.03 .2015$ 